\title{
Conceptual design of an off-momentum collimation system in the CERN Super Proton Synchrotron for High-Luminosity Large Hadron Collider proton beams
}

\author{
Marcin Patecki@, ${ }^{*} \dagger$ Alessio Mereghetti®, ${ }^{\dagger}$ Daniele Mirarchi®, and Stefano Redaelli \\ European Organization for Nuclear Research (CERN), CH-1211 Geneva 23, Switzerland
}

(Received 21 January 2021; accepted 26 July 2021; published 10 September 2021; corrected 28 September 2021)

The CERN accelerator complex is undergoing an upgrade in order to meet the requirements of the High-Luminosity Large Hadron Collider (HL-LHC). One of the key needs is to increase the intensity of beams injected into the LHC from the current $1.15 \times 10^{11}$ to $2.3 \times 10^{11} \mathrm{p}^{+} /$bunch. This requires a beam intensity at injection in the Super Proton Synchrotron (SPS), the last injector before the LHC, as high as $2.6 \times 10^{11} \mathrm{p}^{+} /$bunch, given that a budget for losses of about $10 \%$ is included in the design. However, previous experience with high intensity beams suggests that the total amount of losses in the SPS can be even higher, causing an increased activation, faster aging, and consequent early failure of machine equipment, in addition to an increase in the requirements for preinjectors in order to still meet the HL-LHC target in spite of higher losses. In this paper, we propose a collimation system to be used with HL-LHC proton beams in the SPS in order to intercept and safely dispose of beam losses in ad hoc locations and therefore provide the machine with protection against activation and equipment aging. The design is based on a two-stage concept, where the primary stage intercepts particles that otherwise would be lost, and the secondary stage employing an absorber where lost particles are finally disposed of. Numerical simulations prove a cleaning efficiency of the proposed system of at least $80 \%$, substantially reducing the spreading of particle losses around the machine. Furthermore, the performance of the collimation system has a low sensitivity to common machine errors, and the design is cost efficient, with minimal hardware changes.

DOI: 10.1103/PhysRevAccelBeams.24.093002

\section{INTRODUCTION}

The CERN Super Proton Synchrotron (SPS) [1] is the last accelerator in the injection chain of the Large Hadron Collider (LHC) [2]. It is undergoing an upgrade in the framework of the LHC Injectors Upgrade (LIU) Project [3] to meet the challenging parameters of future HighLuminosity LHC (HL-LHC) [4] proton beams. To achieve the luminosity required by the HL-LHC, the SPS bunch intensity at injection will be nearly doubled to up to $2.6 \times 10^{11} \mathrm{p}^{+} /$bunch with a budget for particle losses of $10 \%$ to guarantee $2.3 \times 10^{11} \mathrm{p}^{+} /$bunch at extraction [3]. A few $\%$ of the losses are associated with scraping at the flattop, while the rest of the budget $(6 \%-7 \%)$ is taken by losses at the injection, flat bottom and start of the

\footnotetext{
"marcin.patecki@cern.ch

Present address: Warsaw University of Technology, Faculty of Physics, ul. Koszykowa 75, 00-662 Warsaw, Poland.

Present address: Centro Nazionale di Adroterapia Oncologica, CNAO, Strada Campeggi, 53, 27100 Pavia, Italy.
}

Published by the American Physical Society under the terms of the Creative Commons Attribution 4.0 International license. Further distribution of this work must maintain attribution to the author(s) and the published article's title, journal citation, and DOI. energy ramp. However, measurements done in 2015 with $2 \times 10^{11} \mathrm{p}^{+} /$bunch suggest that the expected losses in the SPS could exceed the $10 \%$ target for HL-LHC beams [5].

Systematically losing a significant fraction of the beam during the HL-LHC beam production in the SPS would lead to an increased activation spread all around the ring, faster aging, and consequent early failure of the equipment, in addition to an increase in the requirements for preinjectors in order to still meet the HL-LHC target in spite of higher losses. In this paper, we propose the design of an offmomentum collimation system to be used with HL-LHC proton beams in the SPS in order to intercept and safely dispose of beam losses in ad hoc locations. At this stage, the proposed design is not a part of the LIU project baseline and it might be implemented in the case of loss issues revealed by the SPS operation that starts in 2021 .

\section{A. SPS beams and optics \\ 1. Proton beams}

The SPS provides protons not only to the LHC, but also to test facilities (e.g., AWAKE [6], HiRadMat [7], etc., ...) and to fixed target (FT) experiments in the North Area. Different beam types are interleaved during the standard SPS operation, meaning that the machine must be quickly 
TABLE I. Comparison of some important parameters of FT and HL-LHC beams in the SPS.

\begin{tabular}{lcccc}
\hline \hline & $\begin{array}{c}\mathrm{p}^{+} \begin{array}{c}\text { injection/extraction } \\
{[\mathrm{GeV} / c]}\end{array} \\
\mathrm{p}^{+} / \text {batch } \\
{\left[10^{13}\right]}\end{array}$ & $\begin{array}{c}\varepsilon_{\text {norm; } x} \\
{[\mu \mathrm{m}]}\end{array}$ & Optics \\
\hline FT & $14 / 400$ & 5.9 & {$[8,12]$} & Q26 \\
HL-LHC & $26 / 450$ & 7.0 & 1.9 & Q20 \\
\hline \hline
\end{tabular}

reconfigurable (in the order of seconds) to meet the requirements of each beam. FT beams, the most frequently used in the SPS, are significantly different from the LHCand HL-LHC-type beams (see Table I). It is important to highlight the large horizontal emittance and lower injection energy of FT beams, resulting in a high horizontal beam size of up to $40 \mathrm{~mm}\left(4 \sigma_{\beta}\right)$ during the injection plateau. A similar beam extension is observed during the slow resonant extraction at $400 \mathrm{GeV}$ due to the beam being set on the third order resonance [8].

FT beams are generated with so-called Q26 optics, whereas LHC beams are presently produced with Q20 optics [9]; the number next to the Q stands for the integer value of tune. The Q20 optics is characterized by a lower transition energy that allows for a larger bunch intensity, but as a consequence the horizontal dispersion function reaches values about a factor of 2 larger than that of the Q26 optics (see Fig. 1), which reduces the available momentum aperture. HL-LHC beams will be produced with the same Q20 optics. Relevant parameters of HL-LHC beams in the SPS, assuming both design and conservative level of losses, are given in Table II. Figure 2 shows the mechanical aperture and three beam envelopes (without the betatron contribution) assuming the rms momentum spread of one standard deviation, the bucket height, and the momentum cut indicate a tight momentum aperture of the machine.

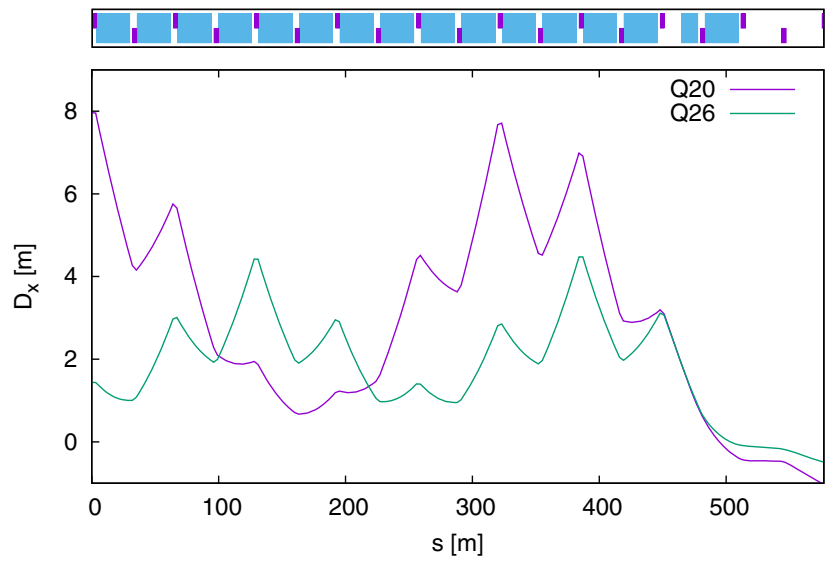

FIG. 1. Horizontal dispersion function for Q20 and Q26 optics. Thanks to the sixfold periodicity of the SPS [1], the horizontal axis includes only half of an arc followed by half of an LSS. The machine layout, comprising dipoles (blue) and quadrupoles (purple), is given in the top panel.
TABLE II. Relevant parameters of HL-LHC beams in the SPS $[3,10]$. Parameters depending on the beam energy correspond to the SPS injection energy. The design loss scenario corresponds to the overall budget minus the contribution from scraping at the flattop. The conservative loss scenario is defined based on experience with high intensity beams [11].

\begin{tabular}{|c|c|c|c|}
\hline $\mathrm{p}^{+}$momentum & $\mathrm{p}$ & {$[\mathrm{GeV} / c]$} & {$[26,450]$} \\
\hline $\begin{array}{l}\text { Normalized transverse } \\
\text { emittance }\end{array}$ & $\varepsilon_{\text {norm } ; x, y}$ & {$[\mu \mathrm{m}]$} & 1.89 \\
\hline Longitudinal emittance & $\varepsilon_{z}$ & {$[\mathrm{eV} \mathrm{s} / b]$} & 0.42 \\
\hline Bucket height (@4.5 MV) & $\delta_{\mathrm{p}, \mathrm{bh}}$ & {$\left[10^{-3}\right]$} & 3.8 \\
\hline Momentum spread & $\sigma_{\delta_{\mathrm{p}}}$ & {$\left[10^{-3}\right]$} & 1.5 \\
\hline Injected intensity & $N_{\mathrm{p}}$ & {$\left[10^{11} \frac{\mathrm{p}^{+}}{\text {bunch }}\right]$} & 2.57 \\
\hline Bunches per train & $N_{b}$ & & $288(4 \times 72)$ \\
\hline $\begin{array}{l}\text { Trains per full LHC } \\
\text { injection }\end{array}$ & & & 20 \\
\hline Train repetition time & & [s] & {$[36,60]$} \\
\hline $\mathrm{p}$ lost per train & Design & $7 \% N_{b} N_{\mathrm{p}}$ & $5.2 \times 10^{12}$ \\
\hline & Conservative & $\mathbf{2 0} \% N_{b} N_{\mathrm{p}}$ & $1.5 \times 10^{13}$ \\
\hline p lost per year & Design & & $1.2 \times 10^{17}$ \\
\hline$(200$ days $/ y \times 120 \mathrm{inj} /$ day $)$ & Conservative & & $3.6 \times 10^{17}$ \\
\hline
\end{tabular}

\section{Heavy ion beams}

About one month per year of LHC operation is usually dedicated to physics with heavy ions (e.g., lead). Lead beams in the SPS are operated at much lower beam intensities (see Table III) compared to proton beams. Therefore, in addition to the shorter total running time for ions, beam losses are less of a concern for the machine where long-term effects are concerned. Hence, there is no need for the proposed collimation system to also be operated with ion beams. The only requirement is to make the collimation system transparent to ion beams, which is easily fulfilled in standard operation. However, reaching

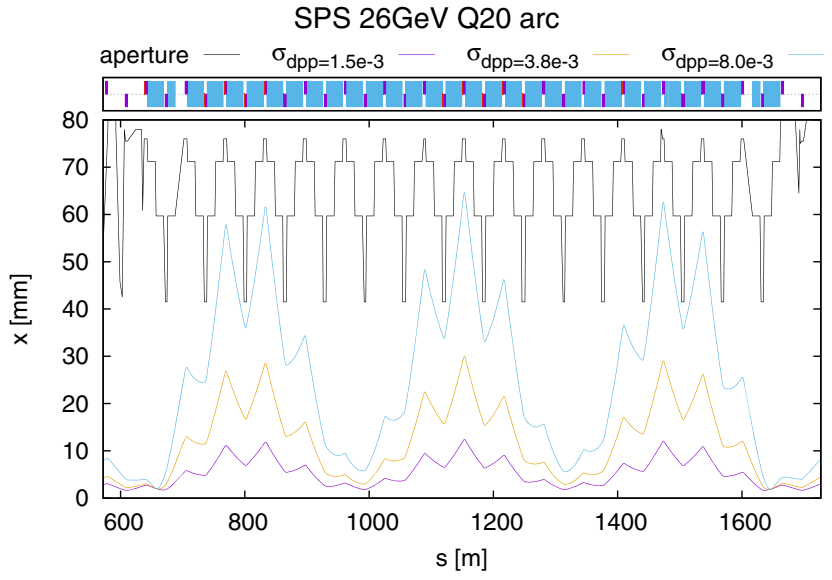

FIG. 2. Momentum beam size calculated as $D_{x} \sigma_{\mathrm{dpp}}$ with three values of momentum offset for the Q20 optics: one standard deviation (purple), bucket height (orange) and momentum cut (blue). Mechanical aperture from the machine model is given in black. 
TABLE III. Relevant baseline parameters of HL-LHC lead ion beams in the SPS $[13,14]$.

\begin{tabular}{lccc}
\hline \hline $\begin{array}{l}\text { Energy injection/extraction } \\
\text { Normalized transverse }\end{array}$ & $\mathrm{E}$ & {$[\mathrm{GeV} / \mathrm{u}]$} & $5.9 / 176.4$ \\
$\quad$ & $\varepsilon_{\mathrm{norm} ; x, y}$ & {$[\mu \mathrm{m}]$} & 1.2 \\
$\quad$ emittance & $\varepsilon_{z}$ & {$[\mathrm{eV} \mathrm{s} / \mathrm{u}]$} & 0.24 \\
Longitudinal emittance & $N_{\mathrm{Pb}}$ & {$\left[10^{8} \mathrm{~Pb} / \mathrm{bunch}\right]$} & 2.1 \\
Bunch intensity & $N_{b}$ & & 48 \\
Number of bunches & $2 \sigma$ & {$\left[10^{-4}\right]$} & 7.0 \\
Momentum spread & & & \\
\hline \hline
\end{tabular}

the HL-LHC luminosity goal with lead ion beams requires a special manipulation of the longitudinal structure of the beam in the SPS, namely slip stacking [12]. During this process, the bunches are combined in the longitudinal plane, which results in significantly larger beam extensions in the dispersive locations of the ring. Even in such a case, a safe margin is expected between the beam and the collimation system. Alternatively, the collimation system can be retracted for ion beam operation.

Table III summarizes the main parameters of HL-LHC lead ion beams in the SPS $[13,14]$.

\section{B. Particle losses in the SPS}

Beam loss mechanisms that occur in the SPS with LHC beams have been extensively studied in recent years and summarized at the SPS Injection Losses Review held in November 2017 [11]. Some updates were also given during the LIU workshop held in February 2019 [15], especially in [16]. A detailed summary of capture and flat bottom losses, including both simulations and measurements, was also published recently in [17]. From these studies, the detailed description of which is beyond the scope of this paper, we have extracted the key conclusions and input them to the collimation system design.

Beam losses occur mostly at beam capture, flat bottom and the start of acceleration due to the longitudinal dynamics of the beam and the reduced mechanical aperture at momentum bottlenecks. This observation determines the main requirement that an off-momentum collimation system is needed, rather than a betatron system.

Losses at beam capture are determined by the longitudinal distribution of the beam received by the SPS from the Proton Synchrotron (PS), the last stage of acceleration before the SPS. In the PS, bunches are operated with a large longitudinal emittance due to longitudinal instabilities [18]. Before being sent to the SPS, the bunches are rotated longitudinally to fit into the SPS rf buckets. The SPS rf buckets are fully filled, with a characteristic S-shape in the initial bunch distribution (see in [17]), which is a consequence of the bunch rotation and resulting in tails extending outside of the bucket area. Particles not captured inside the SPS rf buckets are either immediately lost in the momentum aperture or propagate outside of the main rf buckets (as a coasting beam or satellite bunches) causing losses at a later stage, mostly when the acceleration starts. Protons outside the rf buckets are expected to shift towards negative off-momentum values. It has been demonstrated that the PS bunch tail population is large already before the rotation, which also contributes to losses in the SPS, see [19] for details. Furthermore, as reported in [18], total losses in the SPS related to capture, flat bottom and the start of acceleration strongly increase with larger longitudinal emittance of PS bunches, though with a small effect on flat bottom losses and a more pronounced one on losses at capture and the start of acceleration. A decrease of longitudinal emittance in the PS of about $15 \%$ has been recently explored [16,20], allowing for a potential decrease in losses in the SPS related to uncaptured protons.

The rate of flat bottom losses depends mostly on the SPS bucket area [17], which is controlled by the voltage of the main $200 \mathrm{MHz}$ traveling wave cavities (TWC). For the TWC voltage of $3 \mathrm{MV}$ that corresponds to a smaller than usual bucket area, bunches experience a rapid drop of intensity caused by the limited longitudinal acceptance. A constant rate of intensity decrease is also observed, caused by the limited momentum aperture, and still present even for a smaller than usual area of the buckets. For the alternative scenario, i.e., with the TWC voltage of $7 \mathrm{MV}$ (larger than usual bucket area), more particles are captured within the bunches, but the particle loss rate along the flat bottom is increased by about $50 \%$, resulting in nearly the same beam transmission as for the TWC voltage of $3 \mathrm{MV}$. Therefore, changing the bucket area causes a redistribution of losses along the flat bottom without any significant effect on the total beam transmission. The nominal TWC voltage of $4.5 \mathrm{MV}$ is close to optimal in terms of total losses [17] which is a compromise between these two effects. Furthermore, according to [19], particles with a relative momentum offset of $90 \%$ of the rf bucket height are already expected to eventually fall out of the bucket and contribute to flat bottom losses.

The limited momentum aperture of the SPS is mostly responsible for the flat bottom losses [17]. Measurements of the mechanical aperture performed with local orbit bumps indicate a systematically lower aperture on the negative side of the horizontal axis in the local coordinate system, by about $5 \mathrm{~mm}$ [21]. This is most probably caused by the faulty installation of flanges at the transitions between main dipoles (MBB) and defocusing quadrupoles (QD), as reported in [21]. The proposed solution is to exchange the 25 most limiting flanges during the Long Shutdown 2 (LS2, 2019-2020) and eventually to exchange all faulty flanges; see [22] for details.

An analysis [18] of beam loss monitors signal over cycle duration and ring location indicates that losses occur at momentum bottlenecks located in a few MBB-QD transitions and that the highest loss rate is observed at the start of acceleration. 


\section{Constraints and requirements for the collimation system design}

For a warm machine like the SPS, losses are of concern for the activation induced around the ring and the lifetime of equipment. Hence, numeric simulations (see Sec. ID) are performed to assess whether the integrated losses, accumulated dose, activation, thermomechanical stresses, etc., related to the collimation system remain within acceptable limits while minimizing losses in the machine aperture. No strict requirement on the collimation system performance is defined, so we follow the approach of providing a maximum possible reduction of ring losses with a collimation system as simple and cost efficient as possible, which in practice is a two-stage system. We have observed that reaching the global cleaning efficiency of more than $85 \%$ (the number of protons lost in collimators divided by the total number of protons lost) is challenging with a two-stage collimation system, especially for such a tight momentum aperture of the SPS operated with the Q20 optics. Given the above considerations, we aim at designing a two-stage collimation system reaching more than $80 \%$ of the global cleaning efficiency under conservative assumptions and in the presence of realistic machine errors.

The collimation system should intercept only halo particles without affecting the beam core. To meet this requirement, the minimum opening of the primary collimator is assumed at $4 \sigma_{\beta}+D_{x} \delta_{\mathrm{p}, \mathrm{bh}}$ ("beam stay-clear" region), where $\sigma_{\beta}$ is the betatron amplitude and $\delta_{\mathrm{p}, \text { bh }}$ is the bucket height. It is desirable to install the collimation system in locations with high dispersion to improve intercepting off-momentum protons. Betatron conditions are less relevant in the considered case. Moreover, as discussed in Sec. IA, different beam types might be interleaved within the SPS supercycle. Therefore, the collimation system should provide a good cleaning performance for HL-LHC beams without spoiling other types of beam. Given the short time duration (usually less than a minute) of SPS cycles and knowing that absorbers are usually bulky objects, it is desirable to avoid movements of collimators between cycles as this might be mechanically challenging. Instead, solutions based on magnetic bumps appear to be more flexible, precise and fast.

\section{Simulation setup}

The cleaning performance of the studied collimation systems is evaluated by using the coupling [23] between FLUKA [24,25] and SixTrack [26], where the former simulates the interaction of particles with matter and the latter tracks the particles through the machine model in a symplectic manner. Simulations typically start at the front face of the primary collimator, with $1 \times 10^{5}$ particles hitting the collimator jaw (on the negative side of the horizontal axis in the local coordinate system) with an average impact parameter of $0.1 \pm 0.01 \mu \mathrm{m}$. Such a small impact parameter was chosen arbitrarily as the diffusion speed of halo particles is unknown; however, it is a conservative choice from the point of view of collimation efficiency. It has been verified that larger impact parameters lead to a better cleaning efficiency of the proposed collimation system, meaning that our choice of the impact parameter corresponds to a lower limit of the collimation system performance. The beam halo distribution is constructed such that the horizontal betatron amplitude (radius in the normalized phase space given by the Floquet transformation) follows a double-Gaussian distribution (90\% with $1 \sigma_{\beta_{x}}$ and $10 \%$ with $3 \sigma_{\beta_{x}}$ ) assigning the maximum horizontal betatron extension and the corresponding angle to each particle sampled in such a way. Afterwards, the required $\delta_{\mathrm{p}}$ value is assigned to each particle in order to reach the desired impact parameter via the dispersion term. Vertical positions and angles follow a Gaussian distribution.

The MAD-X code [27] is used to manage the SPS model and to prepare suitable lattice and optics descriptions to be used as input to the tracking studies. The model of the SPS also includes the measured horizontal aperture with expected improvement after LS2 modifications (see Sec. IB).

\section{BASELINE DESIGN OF THE COLLIMATION SYSTEM AND EXPECTED PERFORMANCE}

This section describes in detail the baseline design of the off-momentum system and the expected performance. The design of the off-momentum collimation system is challenging because of a series of hard constraints, as follows: (i) There is no possibility to change the machine optics, neither locally nor globally. The former would have implied dedicated power supplies, significantly increasing the cost of the system. (ii) There is no possibility to change the machine hardware. Since the SPS does not have a dedicated long straight section (LSS) reserved for cleaning and the system has to be inserted into the arc to be effective, the space availability is very limited. (iii) There is a very limited budget for the hardware.

Therefore, it has been necessary to design any new element as short/small as possible and to reuse the already existing hardware as much as possible. The design is proposed for the Q20 optics.

The first, preliminary concept of the SPS collimation system was presented as early as in 2016 [28]. Also, in that case the concept was based on a two-stage system, with a primary and a secondary collimator per plane; each primary collimator was located in the dispersion suppressor upstream of the LSS1 and the secondary collimators in the LSS1. This design is characterized by a global cleaning efficiency of more than $70 \%$, but it has not been further developed as it does not comply with the FT beam requirements; hence, it would require adjusting the collimator gaps between cycles [29], which would pose challenges to the mechanics. 


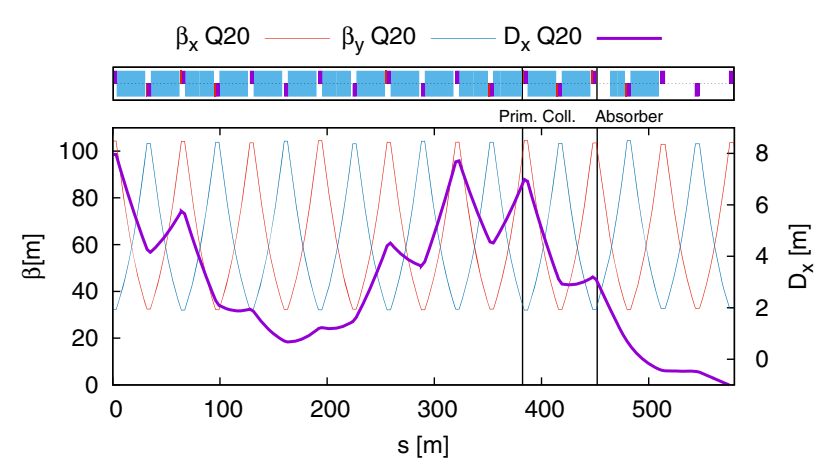

FIG. 3. Optics functions and location of collimators (black vertical lines).

\section{A. System layout}

The proposed design comprises two stages: (i) a primary stage, made of a thin collimator located at the local maximum of dispersion found towards the end of the first arc, i.e., at the short straight section (SSS) of half-cell 112 (see Fig. 3); (ii) a secondary stage, made of a massive absorber located two half cells downstream of the primary stage, in the dispersion suppressor upstream of the LSS1, in the slot of the missed dipole ${ }^{1}$ in half-cell 114, profiting from the existing momentum scraper called TIDP. The name TIDP results from the naming convention used at CERN where T stands for collimator, I stands for internal, D stands for dump and P stands for momentum cleaning.

Since the secondary stage is represented by a massive object, the aperture of which cannot be quickly and precisely reset as appropriate for a machine with multiple types of beams accelerated in a single supercycle as done in the SPS (see Sec. I), an orbit bump is used to steer the beam particles outscattered of the primary stage to the secondary stage.

Figure 3 shows the Q20 optics functions in the region where the collimation system is proposed to be installed; the position of the collimators is highlighted. Table IV shows the optics functions at the two stages. The betatron phase advance between stages is $74^{\circ}$.

The primary collimator is located at a local maximum of dispersion, in order to be an effective momentum aperture bottleneck (a momentum cut of about $1.4 \delta_{\mathrm{p}, \mathrm{bh}}$ ). This aims to intercept beam particles that would otherwise hit the machine aperture (primary halo). Due to space constraints, it can only be a short device, and behave as a scatterer/ scraper. The secondary stage is located in a region of relatively high dispersion, with a favorable phase advance with respect to the primary stage; this is to profit from both the increase in betatron amplitude and the energy loss to intercept protons outscattered by the primary stage (secondary halo). The slot of the missing dipole allows the effective deployment of a massive object, and the existing

\footnotetext{
${ }^{1}$ Dispersion suppression in the SPS is accomplished by means of the "missing dipole" technique.
}

TABLE IV. Linear horizontal optics functions at the primary collimator and absorber (TIDP) for the Q20 optics. Values of a betatron $\sigma$ for a normalized emittance of $1.89 \mu \mathrm{m}$ and the value of the product between the local dispersion function and the bucket height (i.e., $3.8 \times 10^{-3}$ ) are shown as well. The values at the entrance of the devices are reported. $\mathrm{CO}$ and $\mathrm{CO}^{\prime}$ correspond to the closed orbit position and angle.

\begin{tabular}{|c|c|c|}
\hline Parameter & Primary stage & Secondary stage \\
\hline$\beta[\mathrm{m}]$ & 97.5 & 94.1 \\
\hline$\alpha$ & -1.8 & 1.7 \\
\hline $\mathrm{D}[\mathrm{m}]$ & 6.78 & 2.86 \\
\hline D' & 0.10 & -0.09 \\
\hline $\mathrm{CO}[\mathrm{mm}]$ & 0.0 & -17.5 \\
\hline $\mathrm{CO}^{\prime}[\mathrm{mrad}]$ & 0.0 & 417.3 \\
\hline$\sigma_{b}[\mathrm{~mm}]$ & 2.58 & 2.53 \\
\hline$\sigma_{b}^{\prime}[\mu \mathrm{rad}]$ & 54.4 & 53.8 \\
\hline$D_{x} \delta_{\mathrm{p}, \mathrm{bh}}[\mathrm{mm}]$ & 25.8 & 10.9 \\
\hline
\end{tabular}

momentum scraper TIDP has suitable dimensions to behave as a secondary stage, reusing existing hardware.

\section{The primary collimator}

The primary collimator is made of a $5 \mathrm{~mm}$ thick graphite jaw. Graphite has been chosen for its low atomic number, robustness and conductive properties, making it a suitable material for intercepting beam particles. In the considered location, i.e., the SSS 112, there is only about $86 \mathrm{~cm}$ of space available, but it should be enough to equip the machine with a graphite blade, necessary mechanics and vacuum equipment. The primary collimator uses only one jaw located on the low momentum side of the horizontal axis in the local coordinate system.

With a jaw thickness of $5 \mathrm{~mm}$, protons will experience a betatron amplitude increase and an energy loss at a relatively low level, but enough to make them hit the absorber. A longer primary collimator jaw would imply larger scattering amplitudes and energy shifts, increasing the losses immediately downstream of the primary stage and hence decreasing the fraction of protons outscattered from the primary stage and intercepted by the absorber. Figure 4 shows the betatron amplitude increase (due to the horizontal angle change) and the relative momentum decrease experienced by protons passing through the primary collimator. Both the horizontal $\beta$ and dispersion functions grow along the SSS, implying that the first impact of particles on the jaw occurs towards the end of the blade. This causes only a small increase of the betatron amplitude, less than $1 \sigma_{\beta}$ (see the blue curve on the top plot of Fig. 4). Energy loss at first impact is negligible (see the blue curve on the bottom plot of Fig. 4). Therefore, even if the diffusion speed of the beam protons being lost were to be very small, the first interaction with the primary collimator will make them hit the front face of the primary collimator at the following turns. Interaction with a full 

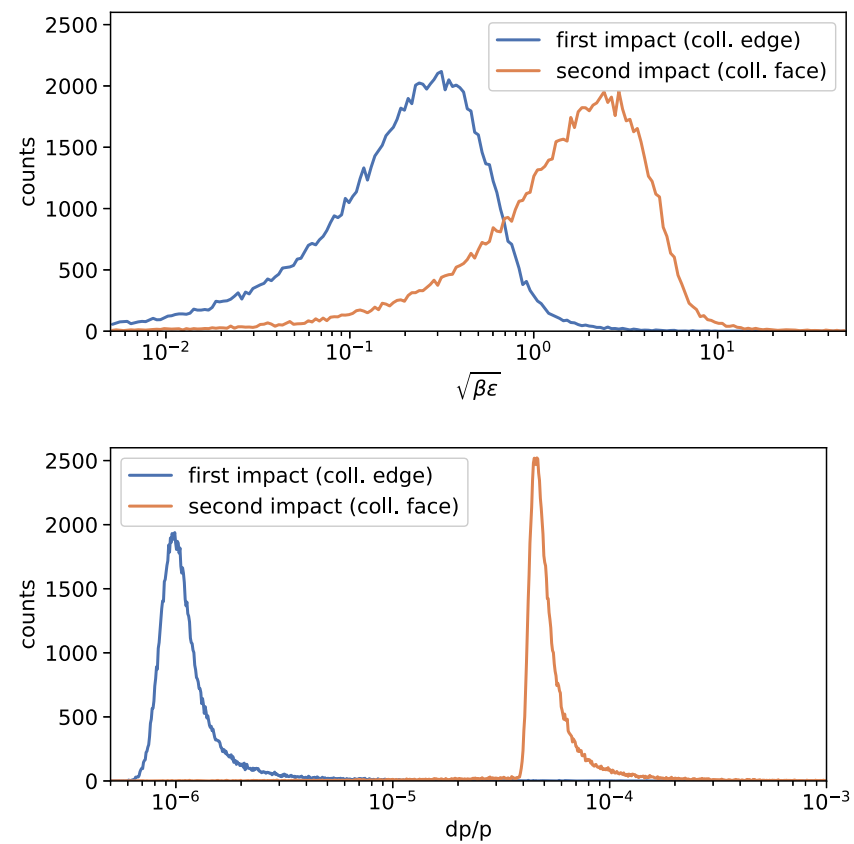

FIG. 4. Histograms of betatron amplitude increase (top) and relative momentum loss (bottom) of protons passing through the primary stage. Please note the logarithmic horizontal scale of both plots.

length of the primary collimator causes a larger increase of betatron amplitude (in most cases this is more than $1 \sigma_{\beta}$ ), which is a dominant process in sending halo particles towards the absorber. Energy loss is a minor effect even for particles experiencing the full length of the primary collimator. Most particles interact with the primary collimator only 2 or 3 times (first hitting its edge and then hitting its front face).

With such a mechanism of beam loss disposal, knowing the diffusion speed experienced by beam protons at the origin of the losses at the flat bottom/start of the ramp is effectively not of utmost importance.

In addition, given the assumptions on the sampled beam (see Sec. ID) which aim at representing an extremely slow diffusion process, the performance of the collimation system is expected to improve with a larger diffusion speed. Similarly, the collimation system performance is expected to improve in the case of losses originating from the injection transient effects - this will result in larger impact parameters; and these in turn lead to a large fraction of halo particles being sent towards the absorber, even after the first interaction with the primary collimator.

\section{The secondary collimator}

As a secondary collimator, the proposal is to reuse an existing absorber (TIDP) [30], originally used with Q26 optics as a momentum scraper [1]. The TIDP core is composed of a $4 \mathrm{~m}$ long block of aluminum, shielded with copper for the last $1.6 \mathrm{~m}$, and surrounded by a $4.3 \mathrm{~m}$ long tank made of cast iron. In regular operations, its horizontal offset from the beam center is adjusted such that the horizontal aperture extends from $-41 \mathrm{~mm}$ to $105 \mathrm{~mm}$ (distances from the machine axis). The horizontal aperture can be adjusted by moving the entire object. The asymmetry in the shift comes from the fact that the TIDP intercepts halo particles with negative values of relative momentum offset $(d p / p)$. The TIDP is optimized for the Q26 optics, as the working point of this optics is close to the one originally foreseen for the SPS [1]. In the case of the Q20 optics, the value of the horizontal dispersion at the TIDP is roughly a factor 2 lower than that at the primary collimator (see Table IV).

Although some adjustments of the TIDP horizontal position are possible, the movement is slow and cannot be applied between cycles; hence, the negative horizontal aperture of the TIDP is kept the same at $-41 \mathrm{~mm}$ throughout the operation, to leave enough space for FT beams (see Sec. IA). Therefore, the optimum retraction of the TIDP with respect to the primary collimator is achieved by using a local three-corrector orbit bump. The selected orbit correctors are MDH.11207 (just downstream of the primary collimator), MDH.11407 (just upstream of the absorber) and MDH.11605 (downstream of the absorber). This allows the creation of an orbit bump that starts downstream of the primary collimator, reaches the maximum extension $(\leq 25 \mathrm{~mm})$ just in front of the absorber and is closed immediately afterwards. In addition, one more corrector, located upstream of the primary collimator (MDH.11007), can be used if it is necessary to operate the primary collimator with larger gaps. The beam envelope at the collimators in cases where the three-corrector orbit bump is used is shown in Fig. 5. It is important to mention that the orbit correctors can efficiently steer the beam to large excursions up to some tens of $\mathrm{mm}$, but only at low beam energies (injection energy and the very beginning of acceleration). Therefore, the collimation system is functional only during the flat bottom and the start of acceleration, which is exactly when the losses occur (see Sec. IB).

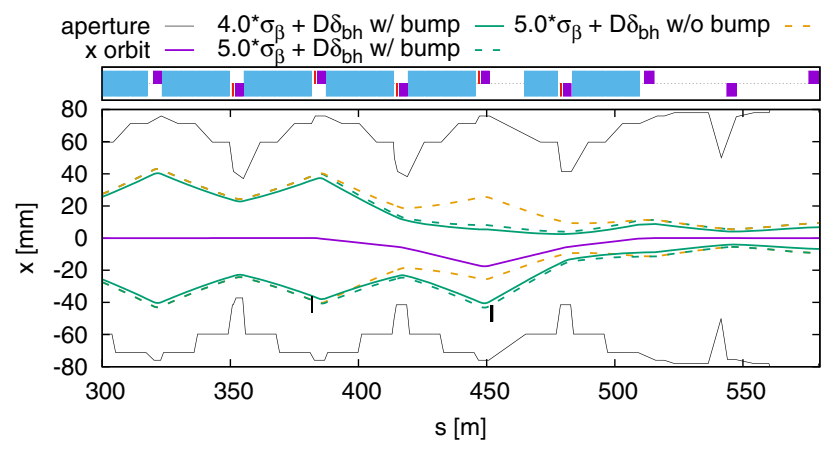

FIG. 5. Orbit bump (purple), beam envelope with (green) and without the orbit bump (orange) at the location of collimators (black rectangles). The machine aperture is given in black and the machine layout is shown in the top panel. 
TABLE V. Operational settings of the baseline design of the SPS off-momentum collimation system. Optics parameters refer to the Q20 optics (see Table IV).

\begin{tabular}{llll}
\hline \hline Stage & Normalized settings & mm settings & \\
\hline Primary & $4 \sigma_{\beta}+D_{x} \delta_{\mathrm{p}, \mathrm{bh}}$ & $-10.3-25.8$ & $=-36.1$ \\
Secondary & $5 \sigma_{\beta}+D_{x} \delta_{\mathrm{p}, \mathrm{bh}}+x_{\text {bump }}$ & $-12.9-10.6-17.5=-41$ \\
\hline \hline
\end{tabular}

The use of a magnetic bump also allows us to control the relative retraction between the stages by changing the orbit bump amplitude at the TIDP. The effect of orbit bump amplitude on cleaning efficiency is presented in Sec. II C 1.

\section{Operational settings}

The design opening of the primary collimator follows the condition $4 \sigma_{\beta}+D_{x} \delta_{\mathrm{p}, \mathrm{bh}}=36.1 \mathrm{~mm}$ to leave enough beam clearance to the beam core. With a $1 \sigma_{\beta}$ relative retraction of the TIDP with respect to the settings of the primary stage, an orbit bump amplitude of about $-17.5 \mathrm{~mm}$ is needed to reach the TIDP aperture at $-41 \mathrm{~mm}$. Normalized and absolute settings of both collimation stages are given in Table V.

The phase space cut corresponding to the chosen operational settings is illustrated in Fig. 6, assuming the expected aperture after the LS2. Due to the lower dispersion, the cut provided by the TIDP is not always in the shadow of that provided by the primary stage, implying that there is a region of the phase space where the hierarchy is not respected, causing a hierarchy breakage. This region of phase space is identified in Fig. 6 by the space outside of the TIDP cut (dashed lines) and inside the cut of the primary stage (solid blue line). Such a region of phase space is reached only by high-amplitude particles (i.e., more than $5 \sigma_{\beta}$ ), which can occur only in the case of instabilities or closed-orbit distortions. It should be noted that the main goal of the off-momentum collimation system is not to protect the machine in these conditions.

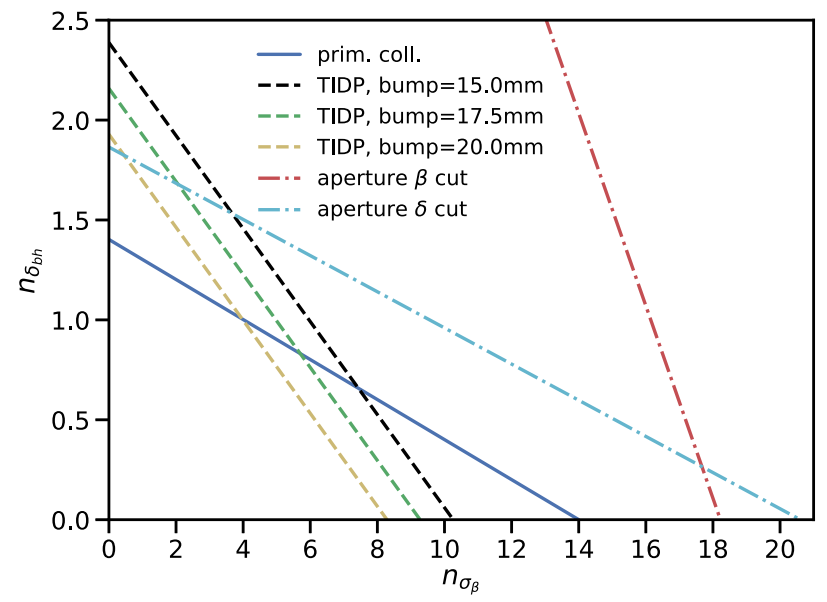

FIG. 6. Cut in phase space provided by the two stages of the baseline proposal of the SPS off-momentum collimation system. The expected post-LS2 aperture has been used.
It will be shown later (see Sec. II B) that the cleaning efficiency stays at an acceptable level even in case of hierarchy breakage without any danger of damaging the collimation system. On the other hand, the TIDP should be robust enough to stand the energy deposition in these conditions; it was found to be the case, even in the more conservative assumptions of full beam impact in a single turn [31].

\section{B. Cleaning performance}

The cleaning performance of the presented design of the SPS off-momentum collimation system is mostly evaluated by means of the global cleaning efficiency, which is the number of protons lost in the collimators $\left(N_{\text {coll }}\right)$ divided by the total losses, i.e., the number of protons lost in the aperture $\left(N_{\text {aper }}\right)$ and in the collimators: $\frac{N_{\text {coll }}}{N_{\text {aper }}+N_{\text {coll }}}$. Another quantity taken into account is the local cleaning inefficiency $(\eta)$, by convention defined as

$$
\eta=\frac{N_{\text {aper }}\left(\Delta s_{1 \rightarrow 2}\right)}{N_{\text {coll }} \Delta s_{1 \rightarrow 2}}
$$

where $N_{\text {aper }}\left(\Delta s_{1 \rightarrow 2}\right)$ is the number of protons lost in the aperture between points 1 and 2 and $\Delta s_{1 \rightarrow 2}$ is the distance between the two points.

\section{Standard operation}

Table VI summarizes the global cleaning efficiency and dominant local losses for the proposed collimation system assuming three sets of aperture limitations: (i) ideal, i.e., as in the MADX model of the SPS; (ii) as measured in 2018 [21]; and (iii) as expected after the LS2 changes [22].

Local cleaning inefficiency plots for these three cases are given in Fig. 7. For the machine with the ideal aperture model, the global cleaning efficiency reaches values well above $80 \%$, with local losses not higher than $1 \%-2 \%$, mostly at some dipoles located close to the collimators. A similar cleaning performance (i.e., only a few percentage points lower) is reached with the aperture model expected after the LS2; however, some local losses of about 2\%-3\% appear at the defocusing quadrupoles, indicating a tight aperture at these locations even after an upgrade of flanges [22]. For the machine with the measured aperture model, the global cleaning efficiency is reduced by a factor of 2 . This happens because a large fraction of the secondary halo is intercepted by momentum aperture bottlenecks located at MBB-QD transitions before being absorbed by the TIDP. The most relevant MBB-QD transitions for the collimation system's cleaning performance are indicated in Fig. 8, where an increase of the global cleaning efficiency after fixing the considered flanges is presented.

The dependence of the cleaning efficiency on the aperture model indicates that having enough margin in phase space between collimator cuts and aperture bottlenecks is crucial 
TABLE VI. Global cleaning efficiency and dominant local losses for three considered aperture models.

\begin{tabular}{llll}
\hline \hline & \multicolumn{3}{c}{ Aperture model } \\
\cline { 2 - 4 } & \multicolumn{1}{c}{ Ideal } & Measured & After LS2 \\
\hline Global cleaning efficiency & $86 \%$ & $42 \%$ & $82 \%$ \\
Dominant local losses & $2 \%($ MBB.11470) & $20 \%($ QD.30110) & $3 \%($ QD.30110) \\
& $1 \%$ (MBB.11490) & $20 \%$ (QD.10110) & $2 \%($ QD.60110) \\
& $1 \%$ (MBA.11230) & $8 \%($ QD.60110) & $2 \%($ MBB.11470) \\
\hline \hline
\end{tabular}

for the collimation system performance. In the unlikely case that the mechanical aperture cannot be corrected (by exchange of faulty flanges), a possible solution is to make the collimator cuts tighter. According to Ref. [19], particles with a relative momentum offset of $90 \%$ of the rf bucket height are expected to eventually fall out of the bucket and contribute to losses. Therefore, the primary collimator half gap can be decreased by about $2.5 \mathrm{~mm}$ (either by mechanically changing the jaw position or by applying an orbit shift at the primary collimator) to match the new condition $4 \sigma_{\beta}+0.9 D_{x} \delta_{\mathrm{p}, \mathrm{bh}}=33.5 \mathrm{~mm}$. The relative retraction of the
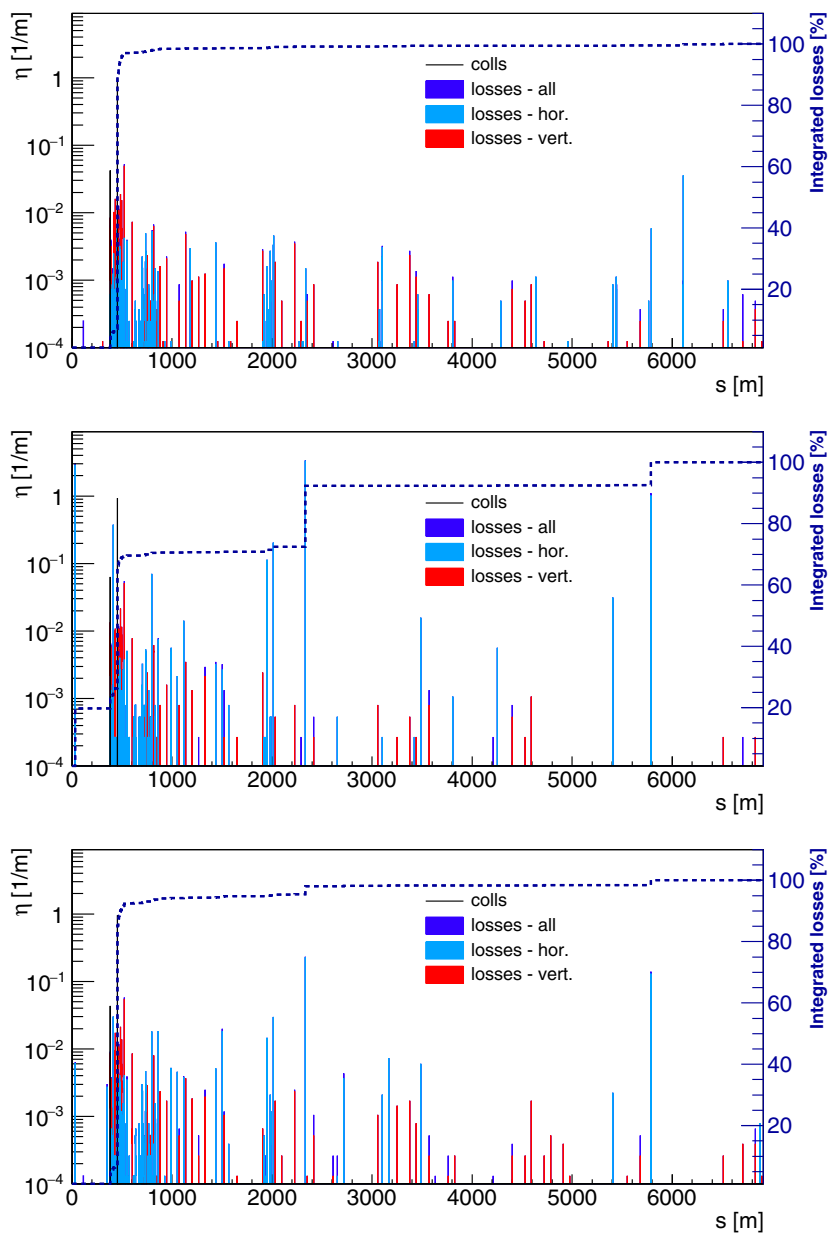

FIG. 7. Local cleaning inefficiency for three aperture models: ideal (top), measured (middle), after LS2 (bottom).
TIDP can be also decreased by $0.5 \sigma_{\beta}$ to set the TIDP cut at $4.5 \sigma_{\beta}+0.9 D_{x} \delta_{\mathrm{p}, \mathrm{bh}}=34.8 \mathrm{~mm}$ resulting in an orbit bump amplitude at the TIDP increased from 17.5 to $19.8 \mathrm{~mm}$. These collimator cuts still fulfill the requirements specified in Sec. IC and allow for the improvement of the global cleaning efficiency from $42 \%$ to $70 \%$ (measured aperture model) with high local losses remaining at MBB-QD transitions: 10\% (QD.30110) and 5\% (QD.60110). The plot of the local cleaning inefficiency for this case is given in Fig. 9. Further improvement of cleaning performance can be obtained by further decreasing the collimator cuts, but the exact settings would have to be verified experimentally so that the total beam transmission is not affected by the collimation system.

\section{Hierarchy breakage}

As shown in Fig. 6, the collimation hierarchy is not respected for high betatron amplitudes (i.e., above $5 \sigma_{\beta_{x}}$ ). Assuming a double Gaussian (i.e., $90 \% 1 \sigma_{\beta_{x}}$ and $10 \% 3 \sigma_{\beta_{x}}$, see Sec. ID) betatron distribution, the collimation hierarchy will be violated by $0.6 \%$ of the considered beam halo population. The effect of the hierarchy breakage on the cleaning efficiency was studied by injecting the concerned particles in front of the TIDP. Following a conservative

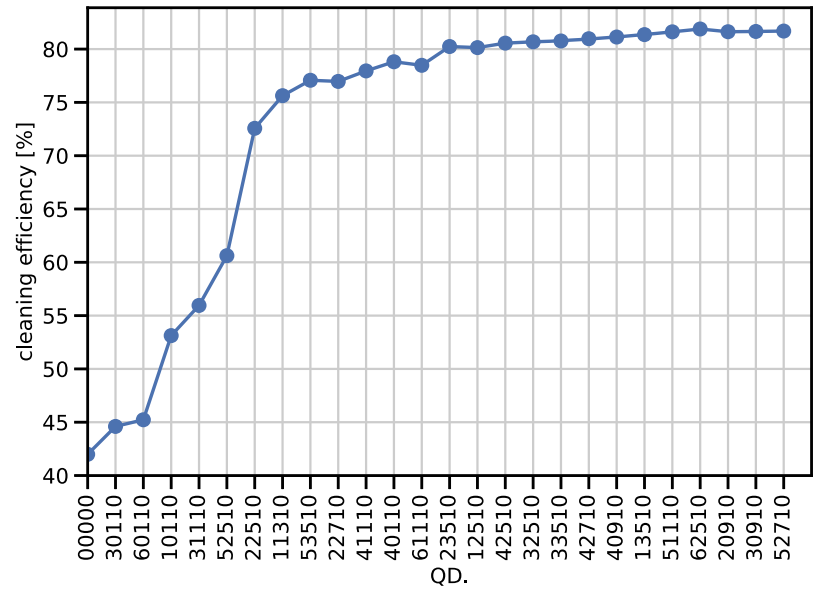

FIG. 8. Global cleaning efficiency after fixing a flange next to a given defocusing quadrupole. For each data point all the flanges to the left of it are also fixed. Data points are joined to guide the eye. 


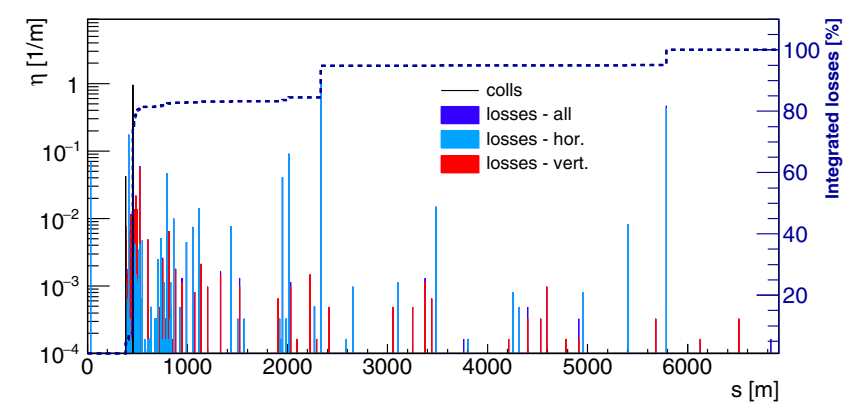

FIG. 9. Local cleaning inefficiency for the measured aperture and with tighter collimation cuts $\left(4 \sigma_{\beta}+0.9 D_{x} \delta_{\mathrm{p}, \mathrm{bh}}\right.$ at the primary stage, $4.5 \sigma_{\beta}+0.9 D_{x} \delta_{\mathrm{p}, \text { bh }}$ at the secondary stage).

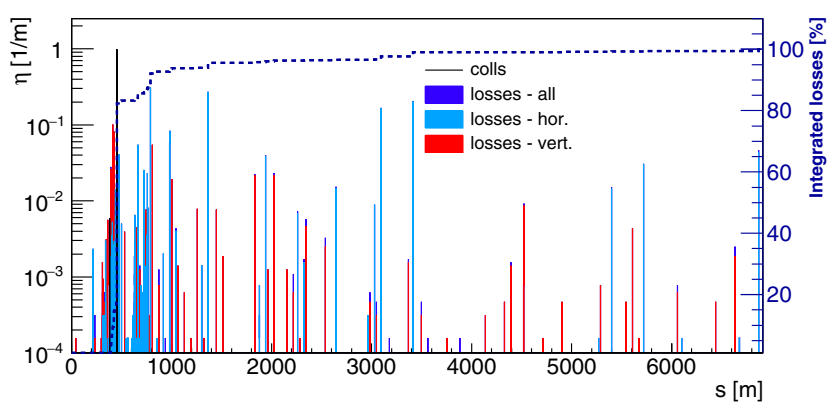

FIG. 10. Local cleaning inefficiency in case of a hierarchy breakage. Aperture model: after LS2.

approach, particles with a very shallow impact parameter were sampled, i.e., along the dashed green line lying under the solid blue line in Fig. 6. In such a case, the global cleaning efficiency reaches more than $60 \%$ (aperture: after LS2), with a few local losses $<5 \%$ in the dipoles located close to the collimators (see Fig. 10). It is important to stress that the hierarchy breakage scenario is not an operational one, it is easy to detect, and its effects can be mitigated either by correcting the beam orbit (either locally at one of the stages or globally), or by further investigation of the machine operation to reveal potential operation issues e.g., an unexpected drift of the orbit, an instability or a tune drift.

\section{Effect of imperfections}

In this section, the sensitivity of the performance of the proposed collimation system to typical machine errors is discussed. The following machine imperfections were identified as possibly affecting the collimation system: error of the orbit bump at the TIDP, a static distortion of the closed orbit throughout the machine, and optics errors (betatron and dispersion beating). All results in this section have been obtained considering the after-LS2 aperture model.

\section{Orbit errors}

The orbit in the SPS is not continuously checked against a reference one and it is not actively corrected by means of an orbit feedback, as is done e.g., in the LHC. On the contrary, closed orbit correction is performed manually by operators only when deemed necessary, until the rms orbit excursion attains the level of a few $\mathrm{mm}$ [32]. When implementing a collimation system, and in particular the one presented here, the closed-orbit correction cannot be applied blindly, but must be done with some care, in order not to degrade the performance of the system or even increase the exposure to a hierarchy breakage. It should be noted that the correct hierarchy is attained with no orbit excursion at the primary stage (the blade still needs to be set at the correct value of $-36 \mathrm{~mm}$ ) and the orbit bump at the TIDP is set at $-17.5 \mathrm{~mm}$ (with the TIDP transverse cut at $-41 \mathrm{~mm}$ ).

Figure 11 shows the dependence of the global cleaning efficiency on the relative retraction between collimation stages, represented by the amplitude of the bump at the TIDP (the closed orbit at the primary collimator is not changed); an error-free machine and after LS2 aperture model are considered. As it can be expected, a smaller bump amplitude corresponds to a larger retraction between stages, resulting in poorer performance of the collimation system. On the other hand, a larger bump amplitude results in a better cleaning performance due to a smaller relative retraction between collimation stages, but it makes the system more sensitive to orbit errors: this can eventually lead to an enhanced breakage of the collimation hierarchy and therefore the degradation of the cleaning performance.

A nonflat global closed orbit may affect the performance of the collimation system if it results in a reduced aperture, especially at momentum bottlenecks. To quantify this effect, we performed a Monte Carlo study on the matching of the closed orbit. We matched the global closed orbit in our model to the same target as used for SPS operation [32], adding the collimation orbit bump on top. In such a case,

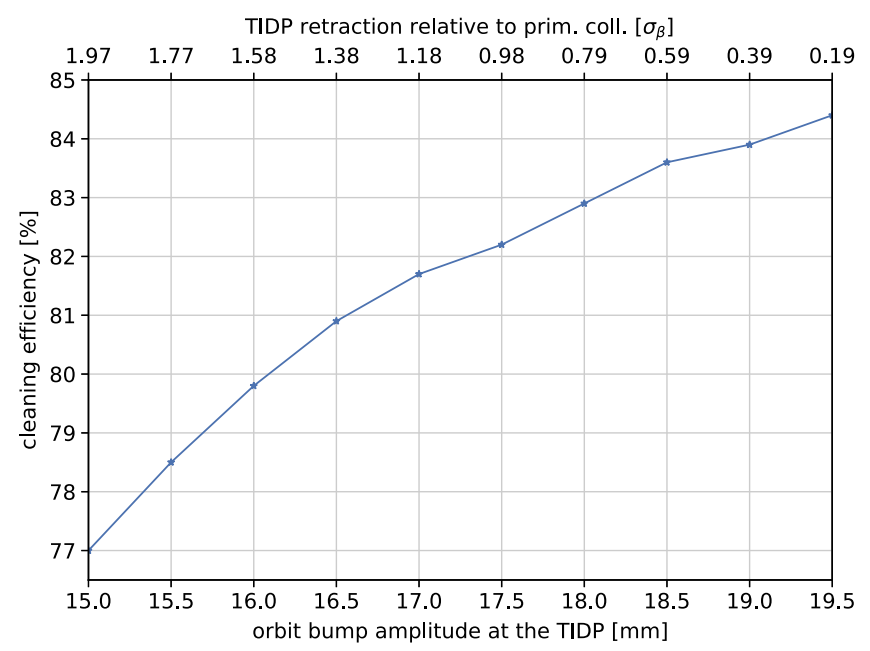

FIG. 11. Global cleaning efficiency as a function of the bump amplitude at the TIDP. Aperture model: after LS2. $17.5 \mathrm{~mm}$ is the baseline value of orbit bump amplitude at the TIDP (see Table V). 
any excursion of the global orbit at the primary collimator is corrected with an orbit corrector located upstream of the primary collimator and the remaining three correctors are adjusted to create a design orbit bump. The Monte Carlo study involved assigning $10 \%$ and $50 \%$ of random Gaussian error to each matching point (given by beam position monitors, BPMs) on approximately 1000 machines. For each machine, the global cleaning efficiency was evaluated. $10 \%$ of random orbit error causes only a systematic drop of the global cleaning efficiency by about 6 percentage points for all generated machines without any significant spread of results. Almost the same average global cleaning efficiency is observed for a random orbit error of $50 \%$ with a bell-shaped spread of results with one standard deviation of 3.4 percentage points. These two observations show that the reference orbit causes a degradation of the global cleaning efficiency, mostly in a systematic manner, with a very weak sensitivity to random errors of the reference orbit. It has been found that the reference orbit reduces the momentum aperture of the machine. A list of unfavorable matching targets and corresponding MBB-QD transitions is given in Table VII. Avoiding negative excursions at these matching targets allows us to recover the expected cleaning performance even in the presence of realistic random errors of the reference orbit.

In conclusion, orbit correction in the SPS in the presence of a collimation system must be performed more carefully than in the past; in particular, the corrected retraction between stages should be kept also after an initial correction, potentially implying resteering the orbit at the collimators. A four-orbit correctors scheme as that outlined in Sec. II A 2 can definitively increase control on the beam closed orbit at the two stages. We suggest developing operation tools for controlling the relative retraction of collimation stages by adapting the orbit correctors strength to the measured closed orbit in the machine, especially taking into account an orbit excursion at both collimators. Operating with an incorrect retraction between stages can be tolerated for a few cycles, but the correct retraction should be finally attained for continuing steady operation. Moreover, we suggest to redefine the reference orbit such that the aperture at momentum bottlenecks is not reduced.

TABLE VII. Unfavorable matching targets of the reference orbit.

\begin{tabular}{lcc}
\hline \hline BPH & $x[\mathrm{~mm}]$ & Affected bottleneck [QD] \\
\hline 11008 & -3.5 & 11110 \\
12608 & -4.7 & 12710 \\
21008 & -3.7 & 21110 \\
23608 & -1.0 & 30110 \\
31008 & -3.6 & 31110 \\
33608 & -4.0 & 40110 \\
53608 & -2.0 & 60110 \\
\hline \hline
\end{tabular}

\section{Optics errors}

Optics errors are introduced in the machine model by randomly applying quadrupole strength errors. About 1000 random machines were simulated, characterized by an rms $\beta$-beating within the $(7,15) \%$ range and an rms D-beating within the $(7,20) \%$ range. Sampled machines with larger beatings were rejected and not simulated. No adjustment of the collimator settings is done in this case. Similarly to the analysis done for closed-orbit errors, the sampled machines were characterized in terms of global cleaning inefficiency; the results are summarized in Fig. 12. One can see that the cleaning performance gets degraded with optics errors for some machines. Figure 13 shows the effect of the $\beta$-beating and the dispersion beating separately. A more pronounced dependence of the cleaning performance on the D-beating is observed, whereas little or no effect from $\beta$-beating is observed.

Therefore, the dispersion has been measured in the SPS [33] in order to quantify its errors actually present in the machine; both raw BPM readout and BPM readout corrected by measured BPM gain errors have been used to reproduce the dispersion [34].

The measured horizontal dispersion based on BPM readouts corrected for gain errors has been imported into the machine model in order to quantify its impact on the cleaning performance. No adjustment of the collimator settings was applied. The global cleaning efficiency reached $85 \%$, even larger than that of an error-free model; this is possible because the measured dispersion is lower at some relevant momentum bottlenecks than that with the ideal dispersion. A very similar result was obtained for the measured horizontal dispersion based on the raw BPM readout, indicating that the estimated performance with random orbit errors was too pessimistic for some machines. This may come from the fact that only an rms dispersion

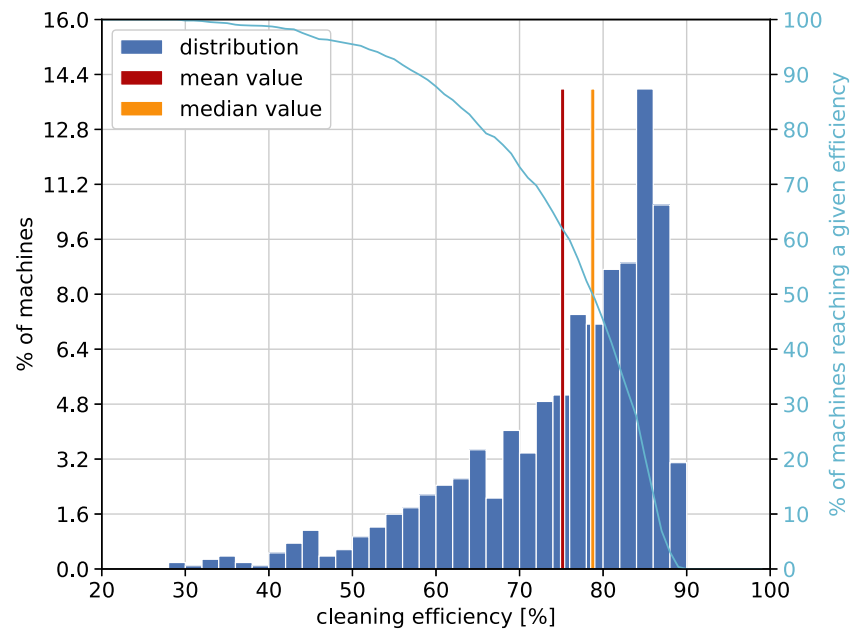

FIG. 12. Global cleaning efficiency distribution for about 1000 random machines with optics errors. 

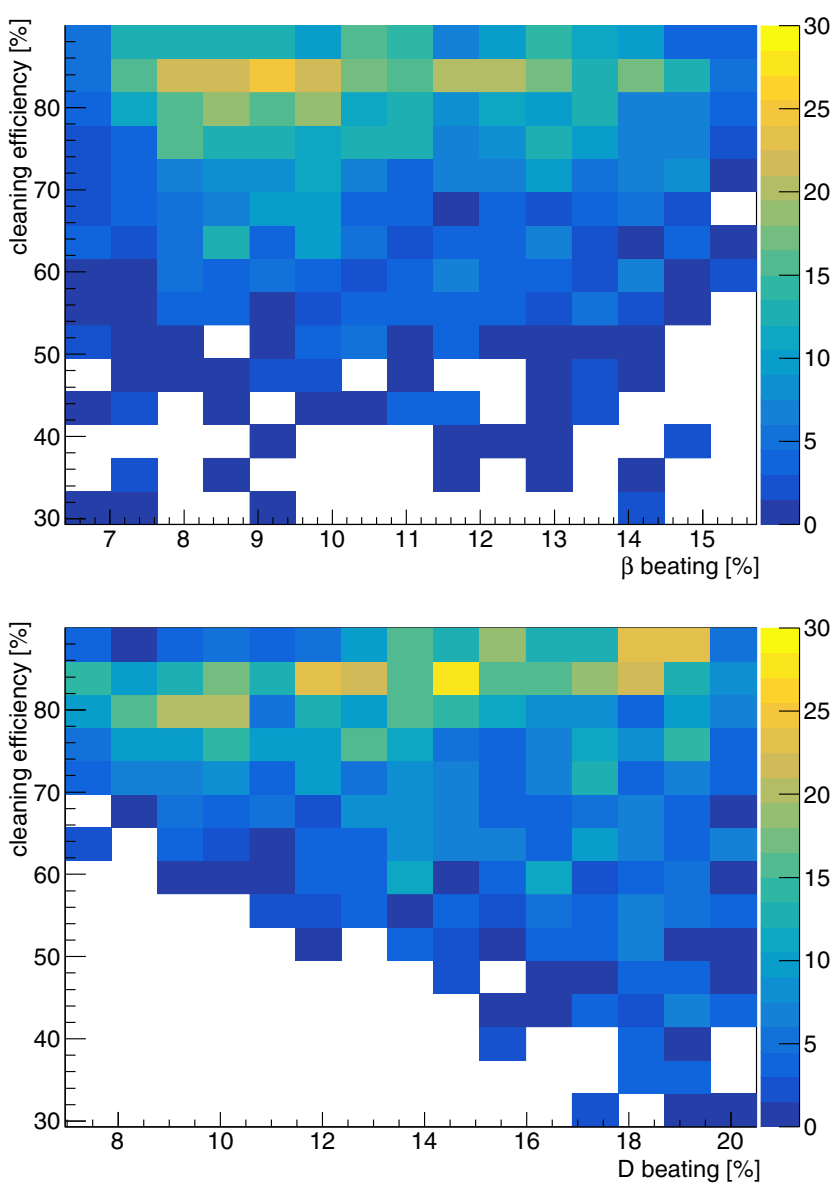

FIG. 13. Distribution of machines attaining a given global cleaning efficiency as a function of $\beta$-beating (top) and D-beating (bottom).

beating was calculated to accept the machine, without checking the local dispersion maxima.

We conclude that optics errors do not affect the cleaning performance of the collimation system if kept within reasonable limits. However, in the case of losses observed at momentum bottlenecks in future operation, it is worth checking the horizontal dispersion of the operated machine.

\section{Combined orbit and optics errors}

Both orbit (with 10\% uncertainty at each matching target) and optics errors were combined in the simulations to check the effect on the cleaning performance. In this case, we assume that the reference orbit avoids negative excursions at momentum bottlenecks and we apply tighter collimator cuts (i.e., $4 \sigma_{\beta}+0.9 D_{x} \delta_{\mathrm{p}, \mathrm{bh}}=33.5 \mathrm{~mm}$ for the primary collimator with $1 \sigma_{\beta}$ relative retraction of the TIDP) in order to have more margin in case of large local dispersion maxima. The global cleaning performance distribution over approximately 2000 randomly generated machines is given in Fig. 14. An average cleaning efficiency of more than $80 \%$ with a few $\%$ of spread indicates that the combination of the considered errors does not affect

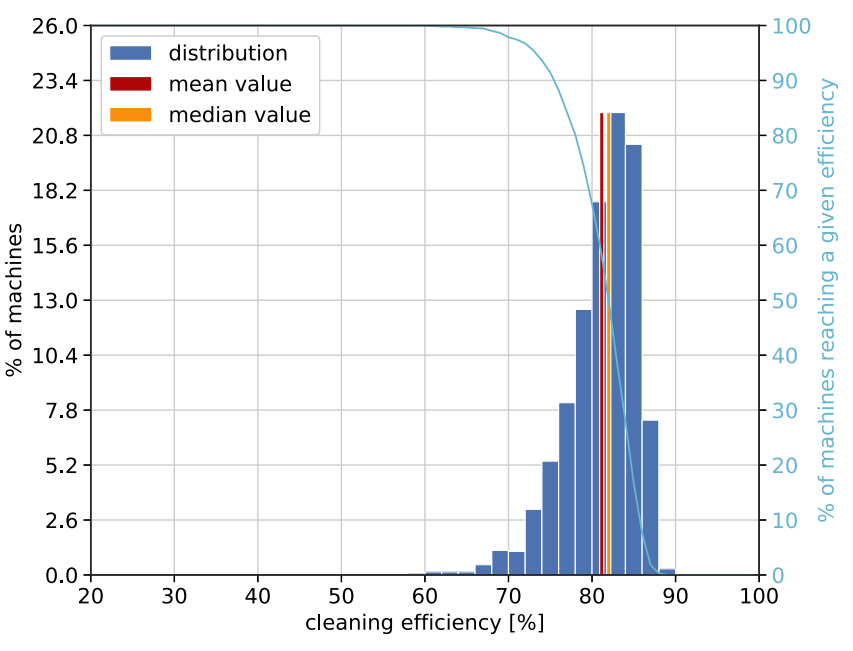

FIG. 14. Global cleaning efficiency distribution for about 2000 random machines with combined orbit and optics errors.

the collimation system's cleaning performance. It should be taken into account that the errors considered in the simulations were rather generous (mostly dispersion), and larger errors are not expected.

\section{Energy deposition, thermomechanical studies and radiation protection considerations}

The presented baseline design has also been analyzed in terms of energy deposition on the concerned devices and sensitive equipment, the thermomechanical response of the collimators, and levels of induced radioactivity with the focus on the regular operation of the system. These studies were made by other members of the LIU-SPS Beam LossProtection-Transfer Lines Working Group, mostly by Esposito (energy deposition), Nuiry (thermomechanical) and Björkman (radiation). We summarize the main conclusions of these studies.

Energy deposition and radiation protection (RP) studies were carried out with FLUKA, loading the map of protons hitting the collimators turn by turn ("touches") as estimated with the SixTrack-FLUKA coupling. The maps of energy deposition estimated with FLUKA were then used for the thermomechanical analyses with finite-element simulation codes.

The peak energy deposition in the primary stage [35] is $\sim 260 \mathrm{~J} / \mathrm{cm}^{3}$ per pulse, corresponding to a peak temperature increase (in adiabatic assumption) of $160^{\circ} \mathrm{C}$ and an integrated dose below 4 GGy per year. The intensity of secondary particle showers developed by the primary stage [35] is almost negligible, since the device is very short ${ }^{2}$; the peak dose in the coils of downstream magnets is below

\footnotetext{
${ }^{2}$ It is $\sim 1 / 100$ of the inelastic scattering length of protons at the SPS injection energy; hence, a very small fraction of protons can undergo an inelastic interaction and hence start a hadronic shower.
} 
$20 \mathrm{kGy} /$ year. No thermomechanical analyses have been performed yet on the primary stage. Similarly, no RP considerations were specifically done on this collimation stage, since the secondary stage, where beam protons are finally disposed of, is of more concern.

The peak energy deposition in the TIDP [10] is $\sim 30 \mathrm{~J} / \mathrm{cm}^{3}$ per pulse, corresponding to a peak temperature increase (in adiabatic assumption) of $14^{\circ} \mathrm{C}$. The intensity of secondary particle showers leaking out of the secondary stage [10] is low, since it is a massive object surrounded by a bulky shielding blanket; the peak dose in the coils of downstream magnets is below $250 \mathrm{kGy} /$ year. Thermomechanical analyses [31] show that the peak temperature reached in the device is $40^{\circ} \mathrm{C}$, assuming constant losses over the injection plateau; Von Mises stresses are relatively low with respect to the yield strength, i.e., $20.5 \mathrm{vs} 240 \mathrm{MPa}$, and the stresses in the steady state are practically negligible. In conclusion, the device is expected to work perfectly well during a regular cleaning operation. The RP group endorses the use of the TIDP as an absorber of proton losses [36], thanks to the high level of selfshielding of the residual dose rates that the present shielding around the device provides. In a case where the TIDP has to be redesigned, it is suggested that the shielding layer is increased by $5 \mathrm{~cm}$, to further improve the residual dose rates near the device.

\section{E. Compatibility with other SPS beams}

While the proposed collimation system has been studied and optimized for HL-LHC beams in the SPS, settings must be chosen such that the system does not interfere with other SPS beams, spoiling their characteristics and creating unnecessary losses for other beam types. As a consequence of the choice to not cycle the primary stage and the existence of other cycles in the SPS supercycle, it is important to check that the other beams have enough clearance. The TIDP already exists in the SPS and does not pose any compatibility problem with SPS beams. Compatibility of the proposed collimation system is therefore only a matter of the primary collimator, the only new device to be installed in the SPS. The nominal half gap of the primary collimator is about $36.1 \mathrm{~mm}$, large enough to stay clear of SPS beams, both for protons and heavy ions (also in the case of the slip-stacking process). In the unlikely case of FT beams reaching a larger extension than the primary collimator half gap (mostly during the resonant extraction process), the primary collimator can be further retracted by a few $\mathrm{mm}$ and reached with the use of a local orbit bump as described in Sec. II A. Beam extensions larger than $40 \mathrm{~mm}$ are not expected at the position of the primary collimator.

\section{F. Effect on machine impedance}

Here we summarize the main factors relevant for the evaluation of the effect of the proposed collimation system on machine impedance. A new device to be added to the machine is the primary collimator, with a jaw length of $5 \mathrm{~mm}$, made of graphite. The distance from the beam center to the primary collimator jaw is about $36 \mathrm{~mm}$. Only one jaw of the primary collimator is needed for operating the collimation system, but there is no obstacle to having two jaws. At the absorber (TIDP), the distance from the beam center to the jaw is about $22.5 \mathrm{~mm}$, but only at the injection energy due to the use of an orbit bump created by orbit correctors of limited strength. After the beam energy ramp, the effect of the TIDP is the same as it would be without the collimation system. The use of an orbit bump increases the asymmetry of the horizontal aperture of the TIDP with respect to beam center by about $17.5 \mathrm{~mm}$.

The impact of the proposed collimation system on beam horizontal stability was calculated by Zannini (CERN Impedance Team) using the TLWALL code [37] with the collimation system included into the SPS impedance model. The resistive wall contribution of the collimation system is dominated by the TIDP, which represents up to $2 \%$ of the total SPS wall impedance [38]. Nearly the same effect is observed when an orbit bump is not present at the TIDP, meaning that the contribution of the collimation system to the machine impedance is negligible.

\section{CONCLUSIONS}

The HL-LHC project aims at increasing the integrated luminosity by a factor of 10 . To meet this goal and deliver beams of the desired brightness, the LIU project has foreseen relevant upgrades of the LHC injection chain, including the SPS, the last accelerator before injection into the LHC. LHC beams in the SPS are affected by losses at injection, at the flat bottom and at the beginning of the ramp. For the HL-LHC a budget for losses of $10 \%$ of the injected beam intensity is assumed in the design. However, experience collected with high intensity beams suggests that the actual amount of losses can be even larger.

Beam loss mechanisms have been thoroughly studied in recent years; their origin has been identified in the longitudinal dynamics of the beam, both in the PS and the SPS. Some of the issues have already been addressed, especially in the PS, or will be mitigated thanks to the SPS $\mathrm{rf}$ system upgrade proposed in the context of the LIU project.

Nevertheless, an off-momentum collimation system is designed as a possible solution for containing losses at the SPS flat bottom in a specific area of the ring and hence limiting the activation and aging of SPS equipment, if losses were still exceeding the allotted budget after the LIU upgrade. At this stage, the proposed design is not a part of the LIU project baseline, but it might be implemented in the case of loss issues revealed by the SPS operation that starts in 2021.

In this paper, we described a proposal of an offmomentum collimation system that intercepts losses of 
the HL-LHC beams in the SPS and reduces the amount of losses distributed over the machine by at least $80 \%$. The presented design consists of a two-stage cleaning system: the primary stage is made of a $5 \mathrm{~mm}$ thick graphite blade installed in the arc at a local peak of dispersion to intercept the off-momentum protons; the secondary stage is realized by reusing the existing momentum scraper TIDP as an absorber for the final disposal of the protons intercepted by the primary collimator. In order to make use of the TIDP, an orbit bump at the secondary stage is necessary. It has been shown that such a design is well compatible with other beams operated in the SPS, opening the possibility of operating the primary collimator at constant settings, with no need to cycle the primary collimator during the SPS supercycle; this simplifies the mechanical design of the device. Moreover, the design is not sensitive to common machine errors, allowing for a reliable protection of the machine. The design is also cost efficient as it requires only the primary collimator to be installed as a new device.

\section{ACKNOWLEDGMENTS}

The study was funded by the LHC Injectors Upgrade project. We also wish to acknowledge the scientific and technical support we received from the LIU team and its working groups, especially the LIU-SPS Beam LossProtection-Transfer Lines Working Group, the SPS Losses and Activation Working Group and the LIU-SPS Beam Dynamics Working Group. Many thanks to V. Kain and H. Bartosik for their support and to L. Esposito, F.-X. Nuiry and D. Björkman for performing the studies reported in Sec. II D and to C. Zannini for his contribution reported in Sec. II F.

[1] The $300 \mathrm{GeV}$ Programme=Le Programme $300 \mathrm{GeV}$ : Two Volumes Bound as One (CERN, Geneva, 1972), https://cds .cern.ch/record/2248363.

[2] O. S. Brüning, P. Collier, P. Lebrun et al., LHC design report, CERN Yellow Reports: Monographs (CERN, Geneva, 2004), https://cds.cern.ch/record/782076.

[3] H. Damerau, A. Funken, R. Garoby et al., LHC injectors upgrade, Technical Design Report, Vol. I: Protons, Technical Report No. CERN-ACC-2014-0337, https://cds.cern .ch/record/1976692.

[4] G. Apollinari, I. B. Alonso, O. Brüning et al., HighLuminosity Large Hadron Collider (HL-LHC): Technical Design Report V. 0.1, CERN Yellow Reports: Monographs (CERN, Geneva, 2017), http://cds.cern.ch/record/ 2284929.

[5] H. Bartosik, G. Iadarola, K. Li et al., Beam dynamics observations of the 2015 high intensity scrubbing runs at the CERN SPS (2016), Report No. CERN-ACC-2016-297, p. 4, MOPOR022, http://cds.cern.ch/record/2207328.

[6] A. Caldwell, AWAKE-A proton driven plasma wakefield acceleration experiment at CERN, Report No. NAPAC2016-TUA2IO01, 2017, TUA2IO01, p. 5, https://cds.cern .ch/record/2314325.
[7] I. Efthymiopoulos, C. Hessler, H. Gaillard et al., HiRadMat: A new irradiation facility for material testing at CERN, Report No. CERN-ATS-2011-232, 2011, p. 4, https://cds .cern.ch/record/1403043.

[8] F. Velotti, W. Bartmann, T. Bohl et al., Characterization of the SPS slow-extraction parameters, Report No. CERNACC-2016-134, 2016, THPOR055, http://cds.cern.ch/ record/2207488.

[9] H. Bartosik, G. Arduini, and Y. Papaphilippou, Optics considerations for lowering transition energy in the SPS, Report No. CERN-ATS-2011-088, 2011, p. 3, https://cds .cern.ch/record/1381216.

[10] L.S. Esposito, Update of FLUKA studies of the SPS offmomentum collimation system, LIU-SPS beam loss, protection and transfer lines WG meeting, 2018, https://indico .cern.ch/event/702596/contributions/2881763.

[11] SPS injection losses review, 2017, https://indico.cern.ch/ event/672967/.

[12] D. Quartullo, T. Argyropoulos, and A. Lasheen, Momentum slip-stacking simulations for CERN SPS ion beams with collective effects, 2018, TUP2WA02, p. 6, http://cds .cern.ch/record/2640826.

[13] J. Coupard, H. Damerau, A. Funken et al., LHC injectors upgrade, Vol. II: Ions, Technical Design Report No. CERN-ACC-2016-0041 (CERN, Geneva, 2016), http://cds.cern.ch/record/2153863.

[14] D. Manglunki, Provisional list of parameters for ions in HL-LHC, EDMS: 1311644, https://edms.cern.ch/ document/1311644.

[15] LHC injectors upgrade workshop, 2019, https://indico.cern .ch/event/774181.

[16] E. Shaposhnikova, Longitudinal beam quality and stability in the SPS (protons and ions), LHC injectors upgrade workshop, 2019, https://indico.cern.ch/event/774181/ timetable/\#17-longitudinal-beam-quality-a.

[17] M. Schwarz, H. Bartosik, E. Shaposhnikova et al., Studies of capture and flat-bottom losses in the SPS, 2018, TUP2WA03, p. 6, http://cds.cern.ch/record/2640827.

[18] H. Bartosik, Overview of measured capture and flat bottom losses, SPS injection losses review 2017, https://indico .cern.ch/event/672967/contributions/2789701/.

[19] A. Lasheen, H. Damerau, J. Repond et al., Improvement of the longitudinal beam transfer from PS to SPS at CERN, Report No. CERN-ACC-2018-173, 2018, THPAF042, p. 4, http://cds.cern.ch/record/2646058.

[20] A. Lasheen, PS intensity reach and longitudinal parameters after LIU, LHC injectors upgrade workshop 2019, https:// indico.cern.ch/event/774181/timetable/\#16-ps-intensityreach-and-long.

[21] V. Kain, SPS momentum aperture and effect on flat bottom losses, SPS injection losses review 2017, https://indico .cern.ch/event/672967/contributions/2789704/.

[22] C. Pasquino and V. Kain, Modification of 25 QD short straight sections configurations in SPS during LS2, Engineering change request, Report No. SPS-VC-EC0004, EDMS: 1946438, https://edms.cern.ch/document/ 1946438.

[23] S. Redaelli, ICFA miniworkshop on tracking for collimation in particle accelerators, 2018, http://cds.cern.ch/ record/2646800. 
[24] A. Ferrari, P. R. Sala, A. Fassò et al., FLUKA: A Multiparticle Transport Code (Program Version 2005), CERN Yellow Reports: Monographs (CERN, Geneva, 2005), http://cds.cern.ch/record/898301.

[25] G. Battistoni, F. Cerutti, A. Fassò, A. Ferrari, S. Muraro, J. Ranft, S. Roesler, and P. R. Sala, The FLUKA code: Description and benchmarking, AIP Conf. Proc. 896, 31 (2007).

[26] G. Ripken and F. Schmidt, A Symplectic Six-Dimensional Thin-Lens Formalism for Tracking (CERN, Geneva, 1995).

[27] H. Grote and F. Schmidt, MAD-X: An upgrade from MAD8, Report No. CERN-AB-2003-024-ABP, 2003p. 4, http://cds.cern.ch/record/618496.

[28] D. Mirarchi, SPS collimation first look and ideas, LIU-SPS beam loss-protection-transfer lines, 2016, https://indico .cern.ch/event/510084/contributions/2032084/.

[29] M. Patecki, Conceptual design of the SPS collimation system, SPS injection losses review 2017, 2018, https:// indico.cern.ch/event/672967/contributions/2789731/.

[30] M. Calviani, Review of SPS beam intercepting devices, SPS losses and activation working group (SLAWG), 2017, https://indico.cern.ch/event/614375/contributions/ $2477457 /$.

[31] J. Maestre and F.-X. Nuiry, TIDP thermomechanical analysis for the HL-LHC and LIU beams, LIU-SPS technical coordination meeting, 2019, https://indico.cern .ch/event/813430/contributions/3392001.

[32] H. Bartosik (private communication).

[33] M. Patecki, Update on LIU SPS collimation studies, LIUSPS beam loss-protection-transfer lines, 2018, https://indico .cern.ch/event/752593/\#25-update-on-liu-sps-collimati.

[34] F. Velotti (private communication).

[35] L.S. Esposito, FLUKA studies of the sps off-momentum collimation system: Scraper + absorber, LIU-SPS beam loss, protection and transfer lines WG meeting, 2018, https://indico.cern.ch/event/702596/contributions/2881763/.

[36] D. Björkman, RP evaluation of collimation setups, LIUSPS beam loss, protection and transfer lines WG meeting, 2018, https://indico.cern.ch/event/736651/contributions/ 3038917.

[37] C. Zannini, Electromagnetic simulation of CERN accelerator components and experimental applications, 2013, https://cds.cern.ch/record/1561199.

[38] C. Zannini (private communication).

Correction: The omission of a secondary affiliation for the second author through a byline footnote has been fixed. 